\title{
Gallery Posets of Supersolvable Arrangements
}

\author{
Thomas McConville|k
}

School of Mathematics, University of Minnesota, Minneapolis, MN 55455, USA

\begin{abstract}
We introduce a poset structure on the reduced galleries in a supersolvable arrangement of hyperplanes. In particular, for Coxeter groups of type A or B, we construct a poset of reduced words for the longest element whose Hasse diagram is the graph of reduced words. Using Rambau's Suspension Lemma, we show that these posets are homotopy equivalent to spheres. We furthermore conjecture that its intervals are either homotopy equivalent to spheres or are contractible. One may view this as a analogue of a result of Edelman and Walker on the homotopy type of intervals of a poset of chambers of a hyperplane arrangement.
\end{abstract}

Résumé. Nous introduisons une structure d'ensemble ordonné sur les galeries réduites dans un arrangement d'hyperplans supersolvable. En particulier, pour les groupes de Coxeter de type A ou B, nous construisons un ensemble ordonné de mots réduits pour l'élément le plus long dont le diagramme de Hasse est le graphe de mots réduits. En utilisant le lemme de suspension de Rambau, nous montrons que ces ensembles ordonnés sont homotopiquement équivalents a des sphères. Nous conjecturons en outre que ses intervalles sont soit homotopiquement équivalents a des sphères ou bien ils sont contractile. On peut considérer cela comme un analogue d'un résultat d'Edelman et Walker sur le type d'homotopie d'intervalles d'un ensemble ordonné des chambres d'un arrangement d'hyperplans.

Keywords: Suspension Lemma, Supersolvable Arrangement, Higher Bruhat, Generalized Baues Problem

\section{Introduction}

This article is about the topology of some posets associated to real hyperplane arrangements. Throughout, the topology attached to a poset $P$ is that of its order complex $\Delta(P)$, the simplicial complex of chains $x_{1}<x_{2}<\cdots<x_{k}$ of $P$. If $P$ is a bounded poset, its proper part $\bar{P}$ is the same poset with those bounds removed.

A reduced gallery of a real central arrangement $\mathcal{A}$ is a sequence of chambers $c_{0}, c_{1}, \ldots, c_{m}$ such that adjacent chambers are separated by exactly one hyperplane, and $c_{0}$ and $c_{m}$ are separated by $m$ hyperplanes. For any codimension 2 subspace $X \in L(\mathcal{A})$, a gallery between opposite chambers $c_{0},-c_{0}$ can cross the hyperplanes containing $X$ in two ways. We introduce a poset $\mathcal{R}\left(\mathcal{A}, r_{0}\right)$ of reduced galleries $r$ ordered by single-step inclusion of sets of codimension 2 subspaces separating $r$ from $r_{0}$. The Hasse diagram of this poset is the usual graph of reduced galleries. An example is shown in Figure 1

\footnotetext{
*Email: mcco0489@umn . edu. Supported by RTG grant NSF/DMS-1148634 

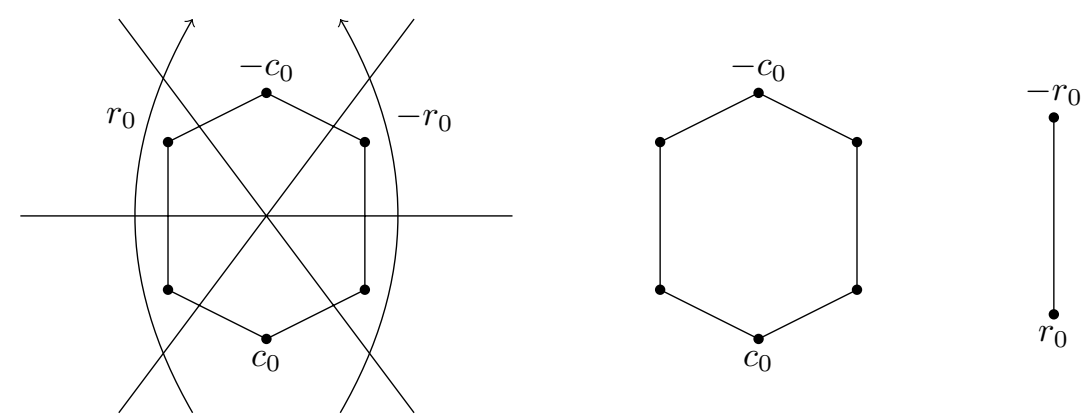

Fig. 1: (Left) An arrangement $\mathcal{A}$ of three lines in $\mathbb{R}^{2}$. (Center) The poset of chambers of $\mathcal{A}$ with base chamber $c_{0}$. (Right) The poset of galleries with base gallery $r_{0}$.

When $\mathcal{A}$ is supersolvable and $r_{0}$ is incident to a modular flag, the poset was essentially shown to be bounded by Reiner and Roichman [10]; see Section 4 for details. In this situation, we prove $\overline{\mathcal{R}}\left(\mathcal{A}, r_{0}\right)$ is homotopy equivalent to a $(\mathrm{rk} \mathcal{A}-3)$-sphere.

For a face $F$ and a chamber $c$ of $\mathcal{A}$, there is a unique chamber $F \circ c$ incident to $F$ that is closest to $c$. A cellular string is a sequence of faces $\left(X_{1}, X_{2}, \ldots, X_{m}\right)$ in $\mathcal{L}(\mathcal{A})$ such that $X_{1} \circ c_{0}=c_{0}, X_{m} \circ\left(-c_{0}\right)=$ $-c_{0}$ and

$$
X_{i} \circ\left(-c_{0}\right)=X_{i+1} \circ c_{0} \quad(\forall i) .
$$

The poset $\omega\left(\mathcal{A}, c_{0}\right)$ of cellular strings is ordered by refinement. Our main result is the following.

Theorem 1.1 Let $\mathcal{A}$ be a real supersolvable hyperplane arrangement with chamber $c_{0}$ and gallery $r_{0}$ both incident to a modular flag. Let $x=\left(X_{1}, X_{2}, \ldots, X_{m}\right) \in \omega\left(\mathcal{A}, c_{0}\right)$ be a cellular string of $\mathcal{A}$. The set of galleries incident to $x$ forms a closed interval of $\mathcal{R}\left(\mathcal{A}, r_{0}\right)$ whose proper part is homotopy equivalent to $\mathbb{S} \sum_{i=1}^{m} \operatorname{codim}\left(X_{i}\right)-m-2$.

We conjecture that all other intervals are contractible.

The motivation for this work primarily comes from two sources: the parameterization of noncontractible intervals in the chamber poset of an arrangement $\mathcal{A}$ proved by Edelman and Walker [7], and a conjecture by Reiner on the noncontractible intervals of the Higher Bruhat orders [11]. We hope to derive new instances of the generalized Baues problem posed by Billera, Kapranov, and Sturmfels via these posets [2]. We summarize the relevant results below.

Two posets naturally arise from a rank $d$ real central hyperplane arrangement $\mathcal{A}$ : the poset of faces $\mathcal{L}(\mathcal{A})$ ordered by reverse inclusion and the poset of chambers $\mathcal{P}\left(\mathcal{A}, c_{0}\right)$ ordered by separation from some base chamber $c_{0}$. These posets are reviewed in Section 3 . Restricting $\mathcal{A}$ to the unit sphere gives a cellular decomposition of $\mathbb{S}^{d-1}$. This implies $\overline{\mathcal{L}}(\mathcal{A})$ is homeomorphic to a $(d-1)$-sphere. In [7], Edelman and Walker use a recursive coatom ordering on an auxiliary poset to prove $\overline{\mathcal{P}}\left(\mathcal{A}, c_{0}\right)$ is homotopy equivalent to $\mathbb{S}^{d-2}$. Moreover, they show the noncontractible open intervals of $\mathcal{P}$ are parameterized by the elements of $\mathcal{L}$.

Theorem 1.2 (Edelman, Walker [7]) Let $\mathcal{A}$ be a real hyperplane arrangement with base chamber $c_{0}$. The set of chambers incident to a face $F \in \mathcal{L}(\mathcal{A})$ forms a closed interval $[c, d]$ in $\mathcal{P}\left(\mathcal{A}, c_{0}\right)$ such that $(c, d)$ is homotopy equivalent to $\mathbb{S}^{\text {codim } F-2}$. Every other open interval of $\mathcal{P}\left(\mathcal{A}, c_{0}\right)$ is contractible 
This theorem establishes an isomorphism between $\mathcal{L}$ and the poset $\operatorname{Int}_{\text {nonc }}(\mathcal{P})$ of noncontractible intervals of $\mathcal{P}$, ordered by inclusion. For an arbitrary bounded poset $P$, the full interval poset $\overline{\operatorname{Int}}(P)$ has a deformation retract to its subposet of proper noncontractible intervals. Furthermore, $\overline{\operatorname{Int}}(P)$ is homeomorphic to the suspension of $\bar{P}$. Thus, EW's theorem may be viewed as an explanation of the homotopy equivalence

$$
\overline{\mathcal{L}}(\mathcal{A}) \simeq \operatorname{susp}\left(\overline{\mathcal{P}}\left(\mathcal{A}, c_{0}\right)\right) .
$$

In [5], Björner applied Theorem 1.2 to compute the homotopy type of a poset $\omega\left(\mathcal{A}, c_{0}\right)$ of cellular strings of $\mathcal{A}$.

Theorem 1.3 (Björner [5]) The poset of cellular strings $\omega\left(\mathcal{A}, c_{0}\right)$ of a rank $d$ arrangement $\mathcal{A}$ with base chamber $c_{0}$ is homotopy equivalent to $\mathbb{S}^{d-2}$.

Björner's proof uses the identification of $\omega\left(\mathcal{A}, c_{0}\right)$ with the poset $\Delta^{H}\left(\mathcal{P}\left(\mathcal{A}, c_{0}\right)\right)$ of chains $\hat{0}=x_{0}<$ $x_{1}<\cdots<x_{m}=\hat{1}$ of $\mathcal{P}$ for which each interval $\left(x_{i}, x_{i+1}\right)$ is noncontractible. Once again a general argument shows that $\Delta^{H}(\mathcal{P})$ is homotopy equivalent to $\Delta(\mathcal{P})$.

Theorem 1.3 as stated above is a special case of the theorem of Billera, Kapranov, and Sturmfels on the homotopy type of a poset of cellular strings in a polytope [2]. However, Björner's argument has a wide-reaching generalization to the cellular string posets of duals of shellable CW-spheres [1].

The paper is structured as follows. The above theorems are interpreted for Coxeter groups in Section 2. Some notation for hyperplane arrangements is given in Section 3. In Section 4 we define the poset of galleries for supersolvable arrangements. In Section 5, we recall the Suspension Lemma and use it to compute the homotopy type of the proper part of $\mathcal{R}$. Theorem 1.1 is proved in Section 6 In Section 7. we draw a parallel between the poset of galleries for supersolvable arrangements and the Higher Bruhat orders. This connection will be developed further in future work.

\section{Coxeter Groups}

A finite Coxeter system $(W, S)$ is a finite group $W$ with a presentation of the form

$$
W=\left\langle S \mid(s t)^{m_{s t}}=1(s, t \in S)\right\rangle
$$

where $m_{s t}=1$ if $s=t$ and $m_{s t}=m_{t s} \in \mathbb{Z}_{\geq 2}$ otherwise. For example, if $S=\left\{s_{1}, \ldots, s_{n-1}\right\}$ with $m_{s_{i}, s_{j}}=3$ when $|i-j|=1$ and $m_{s_{i}, s_{j}}=2$ when $|i-j|>1$, then $W$ is isomorphic to the symmetric group $\mathfrak{S}_{n}$.

A parabolic subsystem $\left(W_{J}, J\right)$ is a subgroup $W_{J}$ generated by a subset $J \subseteq S$. The collection of left cosets of parabolic subgroups

$$
\mathcal{L}(W)=\left\{w W_{J} \mid w \in W, J \subseteq S\right\}
$$

may be viewed as a poset ordered by inclusion.

Let $T=\left\{w s w^{-1} \mid s \in S, w \in W\right\}$ denote the set of reflections of $W$. Define the length $l(w)$ of $w \in W$ to be the smallest value for which $w$ equals $s_{1} \cdots s_{l(w)}$ for some $s_{i} \in S$. The (right) inversion set $\operatorname{Inv}(w)$ of $w \in W$ is $\{t \in T \mid l(w t)<l(w)\}$. The weak Bruhat order $\mathcal{P}(W)$ is the set $W$ ordered by inclusion of inversion sets; see Figure 2 .

An interesting relationship between the posets $\mathcal{P}$ and $\mathcal{L}$ was discovered by Björner. 

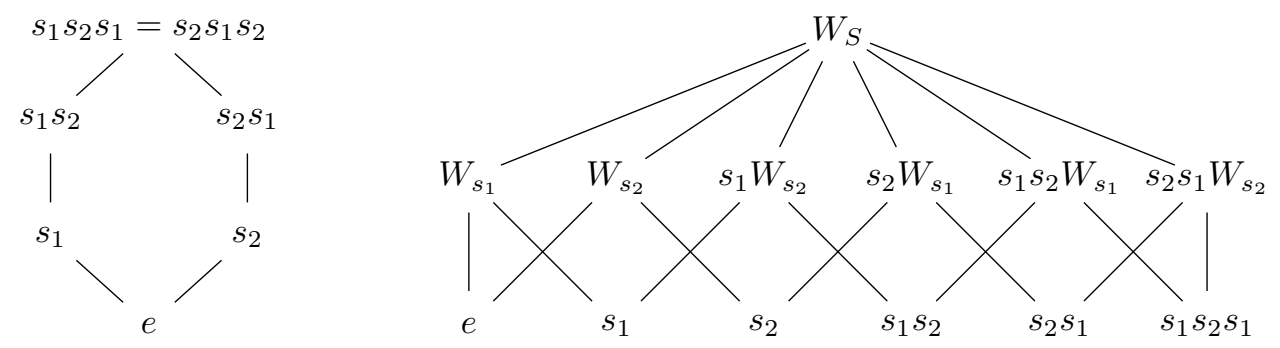

Fig. 2: (Left) The weak order for the type $A_{2}$ Coxeter group. (Right) The parabolic cosets ordered by inclusion.

Theorem 2.1 (Björner [4]) Let $(W, S)$ be a finite Coxeter system. The coset $w W_{J} \in \mathcal{L}(W)$ forms a closed interval $[x, y]$ of $\mathcal{P}(W)$ such that $(x, y)$ is homotopy equivalent to $\mathbb{S}^{|J|-2}$. Every other open interval of $\mathcal{P}(W)$ is contractible.

Any finite Coxeter group has an associated reflection arrangement $\mathcal{A}$ and canonical isomorphisms $\mathcal{L}(\mathcal{A}) \cong \mathcal{L}(W)$ and $\mathcal{P}\left(\mathcal{A}, c_{0}\right) \cong \mathcal{P}(W)$ for any choice of base chamber $c_{0}$. Thus, Theorem 2.1 is a direct consequence of Theorem 1.2

In this article, we describe a lifting of Theorem 2.1 to the intervals of a poset of galleries in a supersolvable hyperplane arrangement. We begin by describing the Coxeter case.

For a finite Coxeter system $(W, S)$ there is a unique element $w_{0} \in W$ with $\operatorname{Inv}\left(w_{0}\right)=T$. Let $\mathcal{R}(W)$ denote the set of reduced words $s_{1} s_{2} \cdots s_{l\left(w_{0}\right)}$ for $w_{0}$. The set $\mathcal{R}(W)$ forms a graph where two reduced words are adjacent if they differ in a single braid move:

$$
s_{1} \cdots s_{k}(\text { stst } \cdots) s_{k+m_{s t}+1} \cdots s_{l} \longleftrightarrow s_{1} \cdots s_{k}(t s t s \cdots) s_{k+m_{s t}+1} \cdots s_{l} \text {. }
$$

The connectivity of $\mathcal{R}(W)$ for arbitrary finite Coxeter groups was originally shown by Tits. Athanasiadis, Edelman, and Reiner showed this graph is $(|S|-1)$-connected in [1].

In [10], Reiner and Roichman give a lower bound for the diameter of $\mathcal{R}(W)$ by defining a set-valued metric on the graph of reduced words. When $W$ is a Coxeter group of type $A$ or $B$ they show this lower bound is achieved by constructing a bounded graded poset whose Hasse diagram is an orientation of $\mathcal{R}(W)$. This construction is recalled in Section 4, see Example 4.1. For the remainder of this article $\mathcal{R}(W)$ will denote this poset.

For $J \subseteq S$, let $w_{0}(J)$ denote the longest element of the subsystem $\left(W_{J}, J\right)$. The poset of cellular strings $\omega(W)$ is the set of words $\left(J_{1}, J_{2}, \ldots, J_{m}\right)$ where $\emptyset \neq J_{i} \subseteq S$ for all $i$ and

$$
w_{0}(S)=w_{0}\left(J_{1}\right) w_{0}\left(J_{2}\right) \cdots w_{0}\left(J_{m}\right), \quad l\left(w_{0}(S)\right)=\sum_{i=1}^{m} l\left(w_{0}\left(J_{i}\right)\right) .
$$

The cellular strings are ordered by refinement, i.e. $\left(I_{1}, \ldots, I_{l}\right) \leq\left(J_{1}, \ldots, J_{m}\right)$ if there is are indices $0=\alpha_{1}<\cdots<\alpha_{t}<l$ such that

$$
w_{0}\left(J_{k}\right)=w_{0}\left(I_{\alpha_{k}+1}\right) w_{0}\left(I_{\alpha_{k}+2}\right) \cdots w_{0}\left(I_{\alpha_{k+1}}\right) \quad l\left(w_{0}\left(J_{k}\right)\right)=\sum_{i=1}^{\alpha_{k+1}-\alpha_{k}} l\left(w_{0}\left(I_{\alpha_{k}+i}\right)\right) .
$$



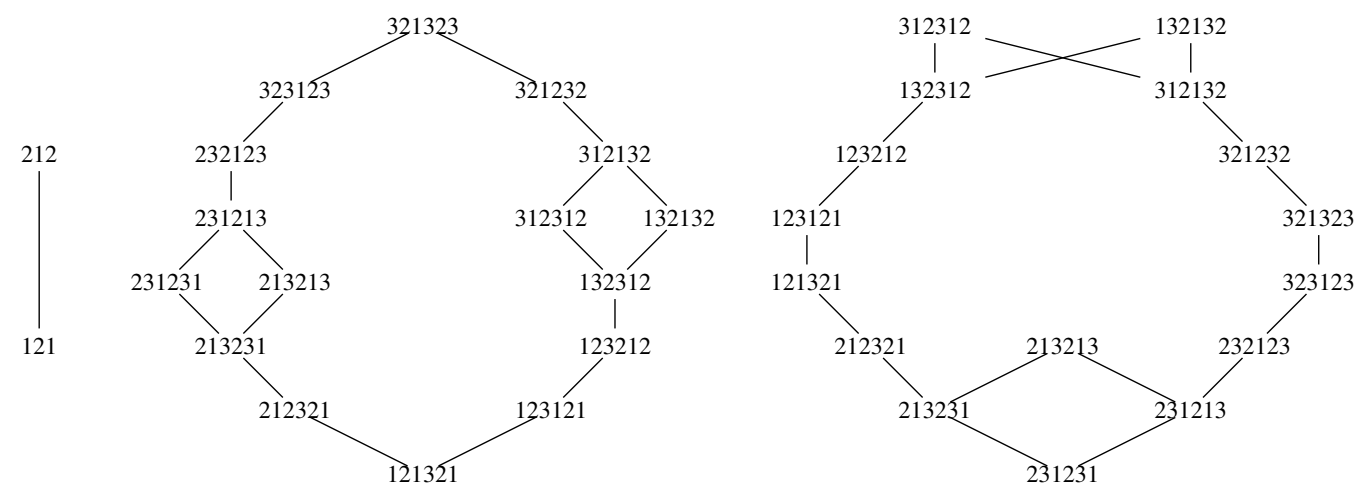

Fig. 3: (Left) $\mathcal{R}(W)$ for type $A_{2}$. (Center) $\mathcal{R}(W)$ for type $A_{3}$. (Right) A bad geodesic order on graph of reduced words for the longest element in type $A_{3}$.

Theorem 2.2 Let $(W, S)$ be a Coxeter system of type $A$ or $B$. The set of reduced words for $w_{0}$ refining a cellular string $\left(J_{1}, \ldots, J_{m}\right)$ forms an interval $[x, y]$ in $\mathcal{R}(W)$ such that $(x, y)$ is homotopy equivalent to $\mathbb{S}^{\sum_{i}\left|J_{i}\right|-m-2}$.

We conjecture that these are the only noncontractible intervals of $\mathcal{R}(W)$.

The weak order on $W$ may be defined by setting $u \leq v$ if there exists a geodesic from $e$ to $v$ passing through $u$ in the Cayley graph of $W$ with respect to the generating set $S$. If the base point in the geodesic order is changed from $e$ to some other element of $W$, an isomorphic poset is obtained since $W$ acts simply transitively on its Cayley graph.

Similarly, $\mathcal{R}(W)$ may be defined by fixing a reduced expression $r_{0}$ and ordering by geodesics in the graph of reduced words for $w_{0}$. However, different choices of $r_{0}$ yield nonisomorphic posets, many of which fail to be bounded or fail to satisfy Theorem 2.2, even in types A and B; see Figure 3 . For type $A$, the base word is

$$
r_{0}=s_{1}\left(s_{2} s_{1}\right)\left(s_{3} s_{2} s_{1}\right) \cdots
$$

while in type $B$, the base is

$$
r_{0}=s_{0}\left(s_{1} s_{0} s_{1}\right)\left(s_{2} s_{1} s_{0} s_{1} s_{2}\right) \cdots .
$$

At this time it is unclear whether a good choice of $r_{0}$ exists for every finite Coxeter group.

\section{Hyperplane Arrangements}

Let $\mathcal{A}$ be an oriented real central hyperplane arrangement in $\mathbb{R}^{d}$. Its lattice of flats $L(\mathcal{A})$ consists of the subspaces $H_{1} \cap \cdots \cap H_{k}$ for $\left\{H_{j}\right\}_{j=1}^{k} \subseteq \mathcal{A}$ ordered by reverse inclusion. The rank $i$ elements $L_{i}(\mathcal{A})$ are the subspaces of codimension $i$.

Each hyperplane $H$ in $\mathcal{A}$ divides $\mathbb{R}^{d}$ into three connected sets $H^{+}, H^{-}, H^{0}$, where $H^{+}$is the positive halfspace defined by $H, H^{-}=-H^{+}$, and $H^{0}=H$. The face poset $\mathcal{L}(\mathcal{A})$ is the set of nonempty cells of the form $\bigcap_{H \in \mathcal{A}} H^{\epsilon(H)}$ where $\epsilon$ is a sign vector in $\{0,+,-\}^{\mathcal{A}}$, ordered by reverse inclusion of their closures. If $F$ is a face defined by $\epsilon_{F} \in\{0,+,-\}^{\mathcal{A}}$, set $F^{0}=\left\{H \in \mathcal{A} \mid \epsilon_{F}(H)=0\right\}$. The map $F \mapsto F^{0}$ 
is an order-preserving map $\mathcal{L}(\mathcal{A}) \rightarrow L(\mathcal{A})$. The set $\mathcal{L}(\mathcal{A})$ is closed under $\circ$ where

$$
\epsilon_{F \circ G}(H)=\left\{\begin{array}{l}
\epsilon_{F}(H) \text { if } \epsilon_{F}(H) \neq 0 \\
\epsilon_{G}(H) \text { else }
\end{array} .\right.
$$

The chambers of $\mathcal{A}$ are the $d$-dimensional faces in $\mathcal{L}(\mathcal{A})$. The $L_{1}$-separation set $L_{1}(c, d)$ of two chambers $c, d$ is the set of hyperplanes separating $c$ and $d$. If $c$ is fixed then $d$ is determined by $L_{1}(c, d)$ by reversing the halfspaces of $L_{1}(c, d)$ in the sign vector for $c$.

Fixing a base chamber $c_{0}$, the poset of chambers $\mathcal{P}\left(\mathcal{A}, c_{0}\right)$ is the set of chambers of $\mathcal{A}$ ordered by $c \leq d$ if $L_{1}\left(c_{0}, c\right) \subseteq L_{1}\left(c_{0}, d\right)$.

\section{Gallery poset}

A gallery is a sequence of chambers $c_{0}, c_{1}, \ldots, c_{m}$ such that

$$
\emptyset=L_{1}\left(c_{0}, c_{0}\right) \subset L_{1}\left(c_{0}, c_{1}\right) \subset L_{1}\left(c_{0}, c_{2}\right) \subset \cdots \subset L_{1}\left(c_{0}, c_{m}\right) \quad\left|L_{1}\left(c_{0}, c_{i}\right) \backslash L_{1}\left(c_{0}, c_{i-1}\right)\right|=1 .
$$

Unless otherwise specified, we will assume the gallery connects an antipodal pair of chambers $c_{0},-c_{0}$. For a fixed base chamber $c_{0}$, a gallery is determined by the order in which the hyperplanes are crossed. The set of galleries admits a free involution $r \mapsto-r$ when $|\mathcal{A}|>1$, where $-r$ is the gallery from $c_{0}$ to $-c_{0}$ which crosses the hyperplanes of $\mathcal{A}$ in the reverse order of $r$.

For a subspace $X \in L(\mathcal{A})$, the localization $\mathcal{A}_{X}$ of $\mathcal{A}$ at $X$ is $\{H \in \mathcal{A} \mid H \supseteq X\}$. If $c$ is a chamber of $\mathcal{A}$, set $c_{X}$ to be the chamber of $\mathcal{A}_{X}$ containing $c$. If $r=c_{0}, c_{1}, \ldots,-c_{0}$ is a gallery, then $r_{X}$ is the sequence $\left(c_{0}\right)_{X},\left(c_{1}\right)_{X}, \ldots,\left(-c_{0}\right)_{X}$ with repetitions removed, which is a gallery of $\mathcal{A}_{X}$.

The $L_{2}$-separation set $L_{2}\left(r, r^{\prime}\right)$ is $\left\{X \in L_{2}(\mathcal{A}) \mid r_{X} \neq r_{X}^{\prime}\right\}$. Given a base gallery $r_{0}, \mathcal{R}\left(\mathcal{A}, r_{0}\right)$ is the poset of galleries between $c_{0}$ and $-c_{0}$ ordered by single-step inclusion of the sets $L_{2}\left(r_{0}, \cdot\right)$. That is, $r \leq r^{\prime}$ if there exists a sequence $r=r_{1}, r_{2}, \ldots, r_{m}=r^{\prime}$ such that

$$
L_{2}\left(r_{0}, r_{1}\right) \subset L_{2}\left(r_{0}, r_{2}\right) \subset \cdots \subset L_{2}\left(r_{0}, r_{m}\right) \quad\left|L_{2}\left(r_{0}, r_{i}\right) \backslash L_{2}\left(r_{0}, r_{i-1}\right)\right|=1 .
$$

Any gallery $r$ is determined by its $L_{2}$-separation set $L_{2}\left(r_{0}, r\right)$ from some fixed gallery $r_{0}$. This follows since the relative order of any two hyperplanes $H, H^{\prime}$ in the total order on $\mathcal{A}$ induced by $r$ is the same as their relative order in $r_{H \cap H^{\prime}}$.

The Hasse diagram of $\mathcal{R}\left(\mathcal{A}, r_{0}\right)$ is an orientation of the graph of reduced galleries from $c_{0}$ to $-c_{0}$ in $\mathcal{A}$. One of the reasons for studying the poset $\mathcal{R}$ is to deduce properties of this graph from those of $\mathcal{R}$.

A flat $X \in L(\mathcal{A})$ of a hyperplane arrangement $\mathcal{A}$ is modular if $X+Y \in L(\mathcal{A})$ for all $Y \in L(\mathcal{A})$. A hyperplane arrangement $\mathcal{A}$ is supersolvable if its lattice of flats $L(\mathcal{A})$ contains a maximal chain of modular elements $\mathbb{R}^{d}=X_{0}<X_{1}<\cdots<X_{d}=0, X_{i} \in L_{i}(\mathcal{A})$. For our purposes, it will be useful to define a maximal chain of faces $F_{0}<F_{1}<\cdots<F_{d}=0, F_{i} \in \mathcal{L}_{i}(\mathcal{A})$ to be a modular chain if the flats $F_{i}^{0}$ are all modular.

A gallery $r$ from $c$ to $d$ is incident to a face $F \in \mathcal{L}(\mathcal{A})$ if $r$ contains the chambers $F \circ c$ and $F \circ d$. A gallery is incident to a subspace $X \in L(\mathcal{A})$ if it is incident to some face spanning $X$. We say a gallery $r$ is incident to a modular flag $c_{0}=F_{0}<F_{1}<\cdots<F_{d}=0$ if it contains $F_{i} \circ c_{0}$ and $F_{i} \circ\left(-c_{0}\right)$ for all $i$. Intuitively this means $r$ first crosses $F_{1}$, then wraps around $F_{2}$, then around $F_{3}$, and so on. 
Example 4.1 The type $A_{n-1}$ reflection arrangement $\mathcal{A}$ is the set

$$
\left\{H_{i j}=\operatorname{ker}\left(x_{i}-x_{j}\right) \subset \mathbb{R}^{n}\right\}_{1 \leq i<j \leq n} .
$$

Its intersection lattice $L(\mathcal{A})$ is in natural correspondence with set partitions of $[n]$ :

$$
\left\{B_{1}, B_{2}, \ldots, B_{m}\right\} \longleftrightarrow \bigcap_{k=1}^{m} \bigcap_{\{i, j\} \subset B_{k}} H_{i j} \in L(\mathcal{A}) .
$$

Similarly, the face poset $\mathcal{L}(\mathcal{A})$ is in correspondence with set compositions of $[n]$ :

$$
B=\left(B_{1}, B_{2}, \ldots, B_{m}\right) \longleftrightarrow \bigcap_{1 \leq i<j \leq n} H_{i j}^{\epsilon_{B}(i, j)} \in \mathcal{L}(\mathcal{A}),
$$

where

$$
\epsilon_{B}(i, j)=\left\{\begin{array}{l}
0 \text { if } B(i)=B(j) \\
- \text { if } B(i)<B(j) \\
+ \text { if } B(i)>B(j)
\end{array} .\right.
$$

The face poset $\mathcal{L}(\mathcal{A})$ contains a modular chain

$$
(1,2,3,4, \ldots, n)>(12,3,4, \ldots, n)>(123,4, \ldots, n)>\cdots>(1234 \cdots n)
$$

Any gallery incident to this flag must contain the chambers

$$
(1,2,3,4, \ldots, n),(2,1,3,4, \ldots, n),(3,2,1,4, \ldots, n), \ldots,(n, \ldots, 4,3,2,1) .
$$

It is clear that there is a unique gallery $r_{0}$ containing these chambers: starting from the base chamber $(1,2, \ldots, n)$, the 2 is pushed to the front, then the 3 , then the 4 , etc. In Coxeter notation, $r_{0}$ corresponds to the reduced word $s_{1}\left(s_{2} s_{1}\right)\left(s_{3} s_{2} s_{1}\right) \cdots\left(s_{n-1} s_{n-2} \cdots s_{1}\right)$.

A simple way to show $\mathcal{R}\left(\mathcal{A}, r_{0}\right)$ is bounded and graded by $r \mapsto\left|L_{2}\left(r_{0}, r\right)\right|$ is via wiring diagrams; see Figure 4 Given the wiring diagram corresponding to some gallery $r$, one can remove elements in the $L_{2}$-separation set by slowly "pulling" the $n$-th wire so it crosses all of the other wires at the end. Each triple $\{i j n\}$ or 2-pair $\{i j, k n\}$ involving wire $n$ on which $r_{0}$ and $r$ disagree is resolved by this procedure without affecting other elements of $L_{2}(\mathcal{A})$. Inducting on $n$, we conclude that $r_{0} \leq r$ as desired.

In [10], Reiner and Roichman generalized the idea of "pulling out the $n$-th wire" for arbitrary supersolvable arrangements to prove that $\mathcal{R}\left(\mathcal{A}, r_{0}\right)$ is a bounded, graded poset. The key property of supersolvable arrangements is the linearity of the fibers of the localization map at a modular line, as described in the following Proposition.

Proposition $4.2(R R[10])$ Assume that $l$ is a modular ray in $\mathcal{L}(\mathcal{A})$. Let $c_{l} \in \mathcal{P}\left(\mathcal{A}_{l},\left(c_{0}\right)_{l}\right)$ be a chamber of the localized arrangement $\mathcal{A}_{l}$. Let $\pi: \mathcal{P}\left(\mathcal{A}, c_{0}\right) \rightarrow \mathcal{P}\left(\mathcal{A}_{l},\left(c_{0}\right)_{l}\right)$ be the localization map.

1. There exists a linear order on the fiber $\pi^{-1}\left(c_{l}\right)=\left\{c_{1}, c_{2}, \ldots, c_{t}\right\}$ such that $c_{1}$ is incident to $l$ and the separation sets $L_{1}\left(c_{1}, c_{i}\right)$ are nested:

$$
\emptyset=L_{1}\left(c_{1}, c_{1}\right) \subset L_{1}\left(c_{1}, c_{2}\right) \subset \cdots \subset L_{1}\left(c_{1}, c_{t}\right)=\mathcal{A} \backslash \mathcal{A}_{l}
$$

This induces a linear order $H_{1}, H_{2}, \cdots$ on $\mathcal{A} \backslash \mathcal{A}_{l}$ such that $H_{i}$ is the unique hyperplane separating $c_{i}$ and $c_{i+1}$. 


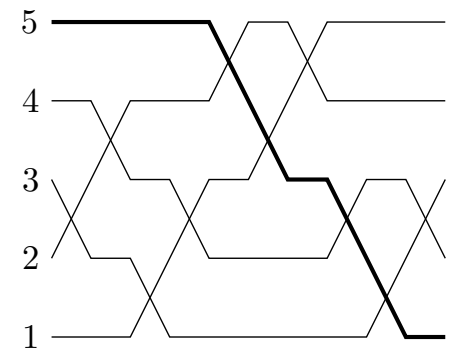

$s_{2} s_{3} s_{1} s_{2} s_{4} s_{3} s_{4} s_{2} s_{1} s_{2}$

23-24-13-14-25-15-12-45-35-34

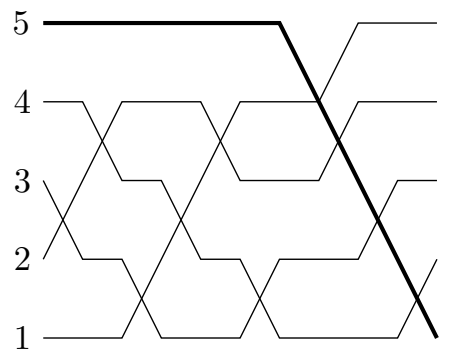

$s_{2} s_{3} s_{1} s_{2} s_{3} s_{1} s_{4} s_{3} s_{2} s_{1}$

23-24-13-14-12-34-15-25-35-45

$\operatorname{Inv}=\{123,124,13 \mid 24\}$

Fig. 4: The inversions in the left wiring diagram containing 5 may be removed by a sequence of elementary flips without introducing new inversions.

2. Using the linear order on $\mathcal{A} \backslash \mathcal{A}_{l}$ from part $(1)$, if $i<j<k$ and if the chamber $c$ incident to $l$ is also incident to $l+H_{i} \cap H_{k}$ then $H_{j} \supseteq H_{i} \cap H_{k}$.

The next proposition says that the fibers of the localization map are bounded.

Proposition 4.3 (RR [10]) Assume that $l$ is a modular ray and let $c_{0}$ be a chamber incident to $l$. Let $\pi$ denote the function $r \mapsto r_{l}$ sending galleries of $\mathcal{A}$ from $c_{0}$ to $-c_{0}$ to galleries of $\mathcal{A}_{l}$. Given a reduced gallery $r_{l} \in \mathcal{A}_{l}$, let $r_{1}$ be the unique gallery incident to $l$ in the fiber $\pi^{-1}\left(r_{l}\right)$ defined by Proposition 4.2 17. If $r_{1} \neq r_{2} \in \pi^{-1}\left(r_{l}\right)$ there exists $X \in L_{2}\left(r_{1}, r_{2}\right)$ incident to $r_{2}$.

Inducting on the rank of $\mathcal{A}$, Reiner and Roichman conclude that if $r_{0}$ is incident to a modular flag of $\mathcal{A}$, then $\mathcal{R}\left(\mathcal{A}, r_{0}\right)$ is a bounded graded poset.

Björner, Edelman, and Ziegler proved that the chamber poset $\mathcal{P}\left(\mathcal{A}, c_{0}\right)$ of a supersolvable arrangement is a lattice if $c_{0}$ is incident to a modular flag [3]. In contrast, the gallery poset $\mathcal{R}\left(\mathcal{A}, r_{0}\right)$ is not a lattice in general; see Example 4.1

The gallery poset $\mathcal{R}\left(\mathcal{A}, r_{0}\right)$ is defined by measuring the "difference" between two galleries with a set $L_{2}\left(r, r^{\prime}\right)$ and ordering the galleries by single-step inclusion of the sets $L_{2}\left(r_{0}, r\right)$. Ordering instead by ordinary inclusion defines a poset $\mathcal{R}_{\subseteq}\left(\mathcal{A}, r_{0}\right)$. We do not know whether these posets are ever distinct when $r_{0}$ is incident to a modular flag of $\mathcal{A}$. When $\mathcal{A}$ is the type $\mathrm{A}$ braid arrangement, this question resembles a question about two versions of the second Higher Bruhat orders, $B(n, 2)$ and $B_{\subseteq}(n, 2)$. These two posets were shown to be the same by Felsner and Weil [8].

\section{Homotopy type of the gallery poset}

Theorem 5.1 Let $r_{0}$ be a gallery incident to a modular flag of a rank $d$ supersolvable arrangement $\mathcal{A}$. The proper part of the poset of reduced galleries $\mathcal{R}\left(\mathcal{A}, r_{0}\right)$ is homotopy equivalent to a $(d-3)$-sphere. 
The main topological result we use to prove the theorem is Rambau's Suspension Lemma [9].

Lemma 5.2 (Rambau) Let $P, Q$ be bounded posets with $\hat{0}_{Q} \neq \hat{1}_{Q}$ and distinguished order ideal $J \subseteq P$. Let $f: P \rightarrow Q$ be a surjective order-preserving map with order-preserving sections $i, j: Q \rightarrow P$. Assume that

1. $i(Q) \subseteq J$ and $j(Q) \subseteq P \backslash J$

2. $(\forall p \in P)(i \circ f)(p) \leq p \leq(j \circ f)(p)$

3. $f^{-1}\left(\hat{0}_{Q}\right) \cap J=\left\{\hat{0}_{P}\right\}$ and $f^{-1}\left(\hat{1}_{Q}\right) \cap(P \backslash J)=\left\{\hat{1}_{P}\right\}$

Then $\bar{P}$ is homotopy equivalent to the suspension of $\bar{Q}$.

Figure 5 shows an application of the Suspension Lemma to the gallery poset of the four coordinate hyperplanes in $\mathbb{R}^{4}$.

Proof: (of theorem) We begin by defining the maps and objects in the Suspension Lemma 5.2 .

Let $\mathcal{F}$ be a modular flag of faces

$$
\mathcal{F}: c_{0}=F_{0}<F_{1}<\cdots<F_{d}=0, F_{i} \in \mathcal{L}(\mathcal{A})
$$

and set $l=F_{d-1}$. Let $r_{0}$ be the unique gallery incident to $\mathcal{F}$.

Define gallery posets $P=\mathcal{R}\left(\mathcal{A}, r_{0}\right), Q=\mathcal{R}\left(\mathcal{A}_{l},\left(r_{0}\right)_{l}\right)$, and let $f: P \rightarrow Q$ be usual localization map removing hyperplanes not containing $l$.

Define a section $i: Q \rightarrow P$ by lifting a gallery $r_{l}$ in $Q$ along $l$ to a gallery from $l \circ c_{0}$ to $l \circ\left(-c_{0}\right)$ and completing it in the unique way described in Proposition 4.2. We similarly define a section $j: Q \rightarrow P$ by lifting along $-l$.

Let $\left(H, H^{\prime}\right)$ be the unique pair of adjacent hyperplanes in $r_{0}$ such that $H \supseteq l$ and $H^{\prime} \nsupseteq l$. We claim that $X_{0}=H \cap H^{\prime}$ is the unique codimension 2 subspace incident to $r_{0}$ not containing $l$. Uniqueness holds since any $Y \in L_{2}(\mathcal{A}) \backslash L_{2}\left(\mathcal{A}_{l}\right)$ contains some hyperplane of $\mathcal{A}_{l}$ by the modularity of $l$, but the hyperplanes in $\mathcal{A}_{l}$ appear in $r_{0}$ before those of $\mathcal{A} \backslash \mathcal{A}_{l}$. Incidence at $X_{0}$ follows from Proposition 4.2 2, Let $J$ denote the order ideal $\left\{r \in P \mid X_{0} \notin L_{2}\left(r_{0}, r\right)\right\}$.

With this setup, we verify the three properties in Lemma 5.2. (1) is clear since $L_{2}\left(r_{0}, i(r)\right)$ is a subset of $L_{2}\left(\mathcal{A}_{l}\right)$ and $L_{2}\left(r_{0}, j(r)\right)$ is a superset of $L_{2}(\mathcal{A}) \backslash L_{2}\left(\mathcal{A}_{l}\right)$. Proposition 4.3 implies $i(r)$ is the minimum element of the fiber $f^{-1}(r)$. Dually, $j(r)$ is the maximum element of $f^{-1}(r)$. This verifies 2 . Finally, (3) follows from the uniqueness of $X_{0}$.

The proof of Theorem 5.1 applies to $\mathcal{R}_{\subseteq}$ with little variation, so we do not repeat it here. An alternate proof using the Crosscut Lemma also computes the homotopy type of $\mathcal{R}_{\subseteq}$, but that argument does not suffice for $\mathcal{R}$.

\section{Main Theorem}

Theorem 6.1 Let $\mathcal{A}$ be a supersolvable arrangement with chamber $c_{0}$ and gallery $r_{0}$ incident to a modular flag. Let $x=\left(X_{1}, X_{2}, \ldots, X_{m}\right) \in \omega\left(\mathcal{A}, c_{0}\right)$ be a cellular string of $\mathcal{A}$. The set of galleries incident to $x$ forms a closed interval of $\mathcal{R}\left(\mathcal{A}, r_{0}\right)$ whose proper part is homotopy equivalent to a sphere of dimension $\sum_{i=1}^{m} \operatorname{codim}\left(X_{i}\right)-m-2$. 

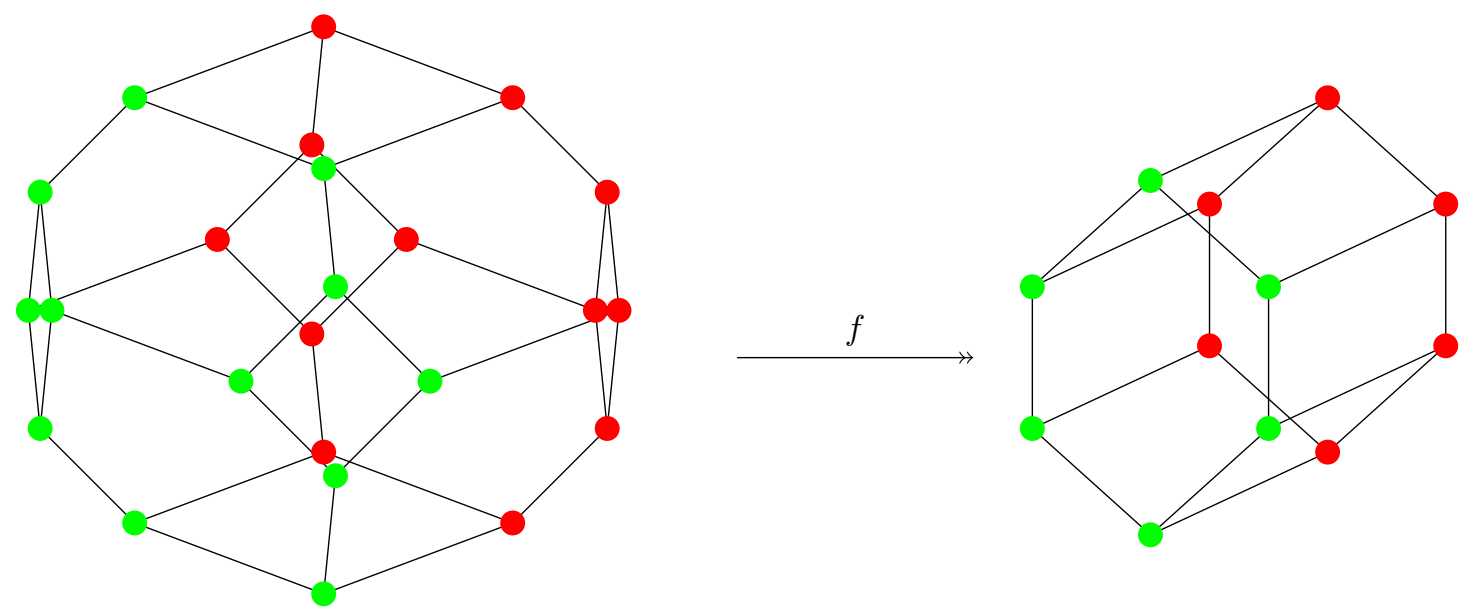

Fig. 5: The suspension lemma applies to this map $\tilde{f}: P \rightarrow(Q \times 2)$ where $P$ and $Q$ are gallery posets of the coordinate hyperplanes in $\mathbb{R}^{4}$ and $\mathbb{R}^{3}$, respectively. The map restricts to the proper parts of $P$ and $Q \times 2$. The fibers $\tilde{f}^{-1}\left(\overline{Q \times 2}_{\geq x}\right)$ are principal upper order ideals, hence contractible. Quillen's fiber lemma (Theorem 10.5 [6]) implies $\bar{P} \simeq \operatorname{susp} \bar{Q}$.

To decompose the set of galleries incident to a cellular string into a product of bounded posets, we make use of the following simple lemma.

Lemma 6.2 Assume $\mathcal{A}$ is supersolvable with modular flag of faces $\mathcal{F}: F_{0}<F_{1}<\cdots<F_{d}=0$. If $X \in L(\mathcal{A})$ then the localized arrangement $\mathcal{A}_{X}$ is supersolvable with modular flag

$$
\mathcal{F}_{X}:\left(F_{0}\right)_{X} \leq\left(F_{1}\right)_{X} \leq \cdots \leq\left(F_{d}\right)_{X}=0
$$

If $r_{0}$ is the unique gallery incident to $\mathcal{F}$, then $\left(r_{0}\right)_{X}$ is the unique gallery incident to $\mathcal{F}_{X}$.

Proof: (of theorem) For each $i, \mathcal{R}_{i}:=\mathcal{R}\left(\mathcal{A}_{X_{i}},\left(r_{0}\right)_{X_{i}}\right)$ is a poset of galleries of a supersolvable arrangement with base gallery incident to a modular flag. By Theorem 5.1 the proper part of $\mathcal{R}_{i}$ is homotopy equivalent to $\mathbb{S}^{\operatorname{codim} X_{i}-3}$.

A gallery $r_{i}$ in $\mathcal{R}_{i}$ can be viewed as a gallery between $X_{i} \circ c_{0}$ and $X_{i} \circ\left(-c_{0}\right)$ in the arrangement $\mathcal{A}$. Since $x$ is a cellular string, any sequence $\left(r_{i} \in \mathcal{R}_{i}\right)_{i}$ can be patched together to a gallery between $c_{0}$ and $-c_{0}$ in $\mathcal{A}$. Consequently, the galleries incident to $x$ may be indentified with the product poset

$$
\mathcal{R}\left(\mathcal{A}_{X_{1}},\left(r_{0}\right)_{X_{1}}\right) \times \cdots \times \mathcal{R}\left(\mathcal{A}_{X_{m}},\left(r_{0}\right)_{X_{m}}\right) .
$$

In general, if bounded posets $P, Q$ are homotopy equivalent to spheres $\mathbb{S}^{p}, \mathbb{S}^{q}$, respectively, then $\overline{P \times Q}$ is homotopy equivalent to $\mathbb{S}^{p+q+2}$. Applying this fact to the above product completes the proof.

Example 6.3 Continuing Example 4.1 the cellular strings $\omega\left(\mathcal{A}, c_{0}\right)$ may be identified with wiring diagrams where multiple wires may cross at a vertical section. The set of galleries incident to a given cellular string $x$ are the simple wiring diagrams obtained by resolving multiple crossings in the diagram associated to $x$. The incident galleries form an interval in $\mathcal{R}\left(\mathcal{A}, r_{0}\right)$ whose minimum element resolves multiple 

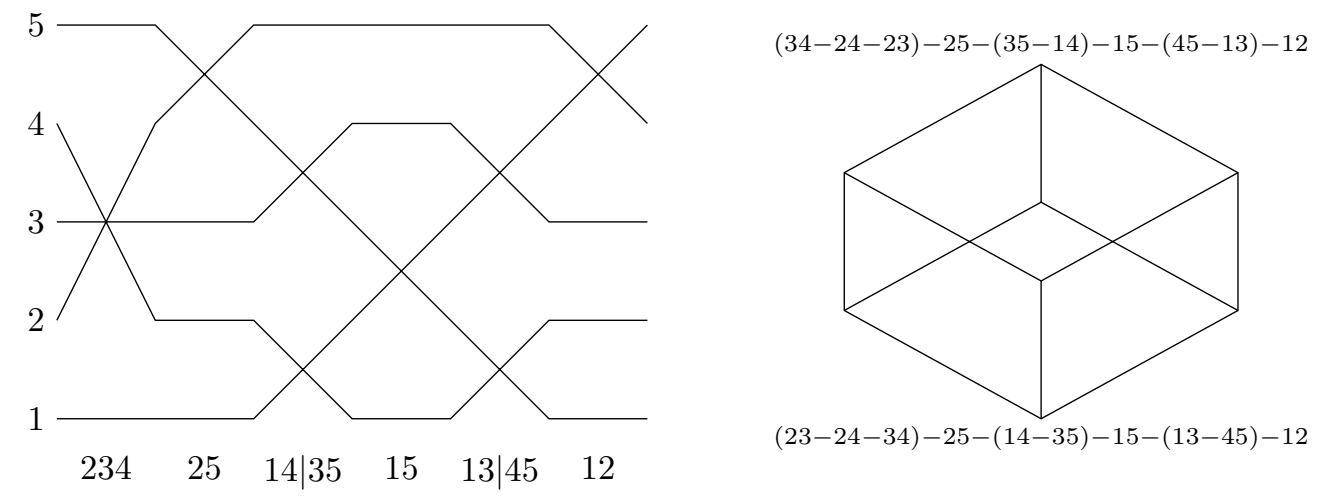

Fig. 6: (Left) A cellular string of the type $A_{4}$ reflection arrangement. (Right) The interval of the gallery poset corresponding to this string. Its proper part is homeomorphic to $\mathbb{S}^{1}$.

crossings in colexicographic order and whose maximum element resolves in reverse colexicographic order. Figure 6 shows an example.

\section{Generic lifting spaces}

The chamber and gallery posets have a common generalization to a poset $G(\mathcal{M}, \mathcal{N})$ of generic singleelement liftings of an oriented matroid. The precise definition is rather technical and will be described in a separate paper. Roughly speaking, $G(\mathcal{M}, \mathcal{N})$ consists of generic affine slices of $\mathcal{M}$ with some prescribed boundary. When $\mathcal{M}$ is realizable as a hyperplane arrangement, the rank 2 slices can be identified with galleries.

Special cases of these posets were called uniform extension posets by Ziegler [13]. Ziegler observed that these posets tend to be poorly behaved. However, when the base extension is an alternating matroid, one obtains a bounded graded poset known as a Higher Bruhat order. We are interested in finding other cases where $G(\mathcal{M}, \mathcal{N})$ is a bounded graded poset with nice topology. We summarize the main results about the Higher Bruhat orders here.

A set family $U \subseteq 2^{\left(\begin{array}{l}{[n]} \\ k+1\end{array}\right)}$ is consistent if for any $(k+2)$-subset $P=\left\{i_{0}<i_{1}<\cdots<i_{k+1}\right\}$ of $[n]$, the intersection $U \cap\left(\begin{array}{c}P \\ k+1\end{array}\right)$ is either $\left\{P \backslash i_{k+1}, P \backslash i_{k}, \ldots, P \backslash i_{t}\right\}$ or $\left\{P \backslash i_{0}, P \backslash i_{1}, \ldots, P \backslash i_{t}\right\}$ for some $t$. The Higher Bruhat order $B(n, k)$ is the collection of consistent set families $U \subseteq 2^{\left(\begin{array}{c}{[n]} \\ k+1\end{array}\right)}$ ordered by single-step inclusion.

In [13], Ziegler proved that $B(n, k)$ is isomorphic a poset of generic single-element liftings of the rank $k$ alternating matroid $\mathcal{M}(n, k)$ on $[n]$. In a way similar to the gallery posets, the single-element liftings are ordered by single-step inclusion of a difference set from the affine alternating matroid $\mathcal{M}(n+1, k+1)$. Ziegler proved that $B(n, k)$ is a bounded, graded poset. He also observed that choosing a different base lifting can determine an unbounded poset. In future work, we explain the relevance of the alternating matroid by showing it is "incident" to a modular flag of $\mathcal{M}(n, n)$.

Rambau showed that $\bar{B}(n, k)$ is homotopy equivalent to $\mathbb{S}^{n-k-2}$ [9]. Analogous to our conjecture on the intervals of the gallery posets, Reiner conjectured that the set of noncontracible intervals of $B(n, k)$ 
is naturally parameterized by the full lifting space $\omega(n, k)$ of $\mathcal{M}(n, k)$ (Conjecture 6.9(a) [11]). Just as in the discussion following Theorem 1.2 , this connection would imply the previous result of Sturmfels and Ziegler that $\bar{\omega}(n, k)$ is homotopy equivalent to $\mathbb{S}^{n-k-1}$ [12].

\section{Acknowledgements}

The author thanks his advisor Pavlo Pylyavskyy and Vic Reiner for helpful discussions and suggestions.

\section{References}

[1] Christos A Athanasiadis, Paul H Edelman, and Victor Reiner. Monotone paths on polytopes. Mathematische Zeitschrift, 235(2):315-334, 2000.

[2] Louis J Billera, Mikhail M Kapranov, and Bernd Sturmfels. Cellular strings on polytopes. Proceedings of the American Mathematical Society, 122(2):549-555, 1994.

[3] A. Björner, P.H. Edelman, and G.M. Ziegler. Hyperplane arrangements with a lattice of regions. Discrete \& computational geometry, 5(3):263-288, 1990.

[4] Anders Björner. Orderings of coxeter groups. Combinatorics and algebra, 34:175-195, 1984.

[5] Anders Björner. Essential chains and homotopy type of posets. Proceedings of the American Mathematical Society, 116(4):1179-1181, 1992.

[6] Anders Björner. Topological methods. handbook of combinatorics, vol. 1, 2, 1819-1872, 1995.

[7] Paul H Edelman and James W Walker. The homotopy type of hyperplane posets. Proceedings of the American Mathematical Society, 94(2):221-225, 1985.

[8] Stefan Felsner and Helmut Weil. A theorem on higher bruhat orders. Discrete \& Computational Geometry, 23(1):121-127, 2000.

[9] Jörg Rambau. A suspension lemma for bounded posets. Journal of Combinatorial Theory, Series A, 80(2):374 - 379, 1997.

[10] Vic Reiner and Yuval Roichman. Diameter of graphs of reduced words and galleries. Trans. Amer. Math. Soc., 365:2779-2802, 2013.

[11] Victor Reiner. The generalized baues problem. New perspectives in algebraic combinatorics, 38:293-336, 1999.

[12] Bernd Sturmfels and Günter M. Ziegler. Extension spaces of oriented matroids. Discrete \& Computational Geometry, 10:23-45, 1993.

[13] Günter M. Ziegler. Higher bruhat orders and cyclic hyperplane arrangements. Topology, 32:259279, 1993. 